\title{
Proposal of a Pretreatment Nomogram for Predicting Local Recurrence after Intensity-Modulated Radiation Therapy in T4 Nasopharyngeal Carcinoma: A Retrospective Review of 415 Chinese Patients
}

\author{
Lu-Lu Zhang, $\mathrm{PhD}{ }^{1}$ \\ Yi-Yang Li, MD² \\ Jiang $\mathrm{Hu}, \mathrm{MD}^{1}$ \\ Guan-Qun Zhou, PhD ${ }^{1}$ \\ Lei Chen, $\mathrm{PhD}^{1}$ \\ Wen-Fei Li, PhD ${ }^{1}$ \\ Ai-Hua Lin, $M D^{3}$ \\ Jun Ma, MD ${ }^{1}$ \\ Zhen-Yu Qi, PhD' \\ Ying Sun, $\mathrm{PhD}$
}

*A list author's affiliations appears at the end of the paper.

Correspondence: Ying Sun, $\mathrm{PhD}$

Department of Radiation Oncology,

Sun Yat-sen University Cancer Center,

State Key Laboratory of Oncology in South China,

Collaborative Innovation Center for

Cancer Medicine, 651 Dongfeng Road East,

Guangzhou 510060, China

Tel: 86-20-87343816

Fax: 86-20-87343295

E-mail: sunying@sysucc.org.cn

Co-correspondence: Zhen-Yu Qi, PhD

Department of Radiation Oncology,

Sun Yat-sen University Cancer Center

State Key Laboratory of Oncology in South China,

Collaborative Innovation Center for

Cancer Medicine, 651 Dongfeng Road East,

Guangzhou 510060, China

Tel: 86-20-87343816

Fax: 86-20-87343295

E-mail: qizhy@sysucc.org.cn

Received July 31, 2017

Accepted November 14, 2017

Published Online November 15, 2017

\begin{abstract}
Purpose
Local relapse-free survival (LRFS) differs widely among patients with T4 category nasopharyngeal carcinoma (NPC). We aimed to build a nomogram incorporating clinicopathological information to predict LRFS in T4 NPC after definitive intensity-modulated radiation therapy (IMRT).
\end{abstract}

\section{Materials and Methods}

Retrospective study of 415 Chinese patients with non-metastatic T4 NPC treated with definitive IMRT with or without chemotherapy at our cancer center between October 2009 and September 2013. The nomogram for LRFS at 3 and 5 years was generated based on multivariate Cox proportional hazards regression, and validated using bootstrap resampling, assessing discriminative performance using the concordance index (C-index) and determining calibration ability via calibration curves.

\section{Results}

Five-year LRFS was $88.8 \%$. We identified and incorporated four independent prognostic factors for LRFS: ethmoid sinus invasion, primary gross tumor volume, age, and pretreatment body mass index. The C-index of the nomogram for local recurrence was 0.732 (95\% confidence interval, 0.726 to 0.738 ), indicating excellent predictive accuracy. The calibration curve revealed excellent agreement between nomogram-predicted and observed LRFS probabilities. Risk subgroups based on total point score cutoff values enabled effective discrimination of LRFS.

\section{Conclusion}

This pretreatment nomogram enables clinicians to accurately predict LRFS in T4 NPC after definitive IMRT, and could help to facilitate personalized patient counselling and treatment strategies.
Key words

Intensity-modulated radiation therapy, Recurrence, Nasopharyngeal carcinoma, Nomograms 


\section{Introduction}

Nasopharyngeal carcinoma (NPC), a malignant tumor that originates in the nasopharyngeal epithelium, is endemic in southern China and Southeast Asia where the age-standardized incidence ranges from 20 to 50 per 100,000 person-years [1]. Radiotherapy is the primary treatment modality for nondisseminated NPC as a result of its complex anatomical location and high radiosensitivity [2,3]. The introduction of intensity-modulated radiation therapy (IMRT) has greatly improved locoregional control in NPC [4]. Recently, Wu et al. [5] reported the 10-year local relapse-free survival (LRFS) rates for patients with $\mathrm{T} 1, \mathrm{~T} 2$, and $\mathrm{T} 3 \mathrm{NPC}$ receiving IMRT were satisfactory $(94.2 \%, 92.5 \%$, and $91.4 \%$, respectively; $\mathrm{p}>$ 0.05); however, the 10-year LRFS rate for T4 NPC remains poor $(79.3 \%, \mathrm{p}<0.05$ vs. T1-T3 NPC). Recurrent NPC remains a challenging clinical problem and traditional management does little to limit local control and, more seriously, frequently induces severe, life-threatening late complications [6]. Thus, it is necessary to develop accurate prognostic models to identify patients with T4 NPC at high risk of local recurrence as early as possible; this may provide an opportunity establish more intensive treatment and follow-up strategies for patients with T4 NPC at high risk of local recurrence.

The American Joint Committee on Cancer (AJCC)/Union for International Cancer Control (UICC) TNM staging system is the most widely used prognostic tool for predicting the risk of recurrence and survival outcomes. Patients with non-metastatic NPC are stratified according to tumor size, invasion and lymph node involvement. However, significant variations in local recurrence after definitive IMRT are observed within each individual T category [5]. This indicates additional, more relevant variables should be integrated to improve prognostication of clinical outcomes for patients with T4 NPC. Previous research demonstrated a number of independent prognostic factors such as such as age, World Health Organization (WHO) histologic type, primary gross tumor volume (GTVp), and pretreatment lactate dehydrogenase (LDH), can significantly predict local recurrence [7-9]. Therefore, a comprehensive tool that estimates individual risk of recurrence by incorporating TNM stage and clinicopathological information, including host factors, treatment strategies, pathological factors, and anatomic factors, could represent a valuable decision-making tool for clinicians.

Nomograms incorporate a variety of important prognostic factors and have been accepted as useful predictive statistical models for quantifying individual risk in several cancers $[10,11]$. Nomograms have been demonstrated to have a better ability to predict prognosis than TNM stage in many types of cancer [10-12]. Hence, the present study aimed to establish an effective prognostic pretreatment nomogram to enable individualized prediction of the risk of local recurrence in patients with T4 NPC treated with IMRT.

\section{Materials and Methods}

\section{Patient selection and staging evaluation}

A total of 415 patients with NPC treated at Sun Yat-sen University Cancer Center (Guangzhou, China) between October 2009 and September 2013 were enrolled in this retrospective study. The eligibility criteria were as follows: (1) newly diagnosed, pathologically proven, previously untreated NPC; (2) no evidence of distant metastasis; (3) stage T4N0-3M0 NPC; (4) treated with IMRT and completed planned radiotherapy with good radiation treatment compliance; and (5) complete clinicopathologic and treatment data available.

Pretreatment staging workup included a complete patient history, physical and neurological examinations, hematology and biochemistry profiles, fiberoptic nasopharyngoscopy, magnetic resonance imaging (MRI) of the nasopharynx and neck, chest radiography, abdominal ultrasonography, and single positron emission tomography computed tomography (PET-CT). Each patient was restaged according to the eighth edition of the AJCC/UICC staging system. Two senior physicians experienced in head and neck cancer (L.C. and W.F.L.) separately evaluated all scans and all disagreements were resolved by consensus.

\section{T classification criteria and stage grouping according to the eighth edition of the AJCC/UICC staging system and proposed staging system}

According to the eighth edition of the AJCC/UICC staging system, tumors confined to the nasopharynx or with extension to the oropharynx and / or nasal cavity without parapharyngeal involvement are classified as T1 disease; tumors with extension to the parapharyngeal space and/or adjacent soft tissue involvement (medial peterygoid, lateral pterygoid, and prevertebral muscle) are classified as T2 disease; tumors with infiltration of bony structures at the skull base, cervical vertebra, pterygoid structures and/or paranasal sinus are classified as T3 disease; tumors with intracranial extension, involvement of the cranial nerves, hypopharynx or orbit or extensive soft tissue involvement (beyond the lateral surface of the lateral pterygoid muscle, parotid gland) are classified as $\mathrm{T} 4$ disease. 
As T4 lesions could have coincidental lesions that should belong to T1-3 categories, we investigated the association between such coincidental lesions and local recurrence in patients with $\mathrm{T} 4$ disease.

\section{Treatment strategies}

During the study period, institutional guidelines recommended concurrent chemoradiotherapy with or without neoadjuvant/adjuvant chemotherapy for stage IVA-IVB. All patients were treated with fractionated IMRT 5 days/wk. Target volumes were contoured according to the institutional treatment protocol [13], in agreement with International Commission on Radiation Units and Measurements Reports 50 and 62 [14]. The prescribed radiation doses were: total of 66-72 Gy at 2.12-2.43 Gy / fraction to the planning target volume (PTV) of the GTVp, 64-70 Gy/28-33 fractions to the involved nodal gross tumor volume PTV, 60-63 Gy / 28-33 fractions to the PTV of CTV-1 (high-risk clinical target volume), and 54-56 Gy/28-33 fractions to the PTV of CTV-2 (low-risk clinical target volumes). A total of $192 / 415$ patients (46.3\%) completed radiotherapy on schedule and 223/415 patients (53.7\%) had prolonged radiotherapy schedules.

Concurrent chemotherapy was $30-40 \mathrm{mg} / \mathrm{m}^{2}$ cisplatin administered weekly or $80-100 \mathrm{mg} / \mathrm{m}^{2}$ cisplatin administered on weeks 1, 4, and 7 of radiotherapy, beginning first day of IMRT. Neoadjuvant chemotherapy was two or three cycles of cisplatin $\left(75 \mathrm{mg} / \mathrm{m}^{2}\right)$ with docetaxel $\left(75 \mathrm{mg} / \mathrm{m}^{2}\right)$; cisplatin $\left(80 \mathrm{mg} / \mathrm{m}^{2}\right)$ with 5 -fluorouracil $\left(1,000 \mathrm{mg} / \mathrm{m}^{2}\right)$; or cisplatin $\left(60 \mathrm{mg} / \mathrm{m}^{2}\right)$ with 5 -fluorouracil $\left(600 \mathrm{mg} / \mathrm{m}^{2}\right)$ and docetaxel $\left(60 \mathrm{mg} / \mathrm{m}^{2}\right)$ every 3 weeks.

\section{Follow-up and end-points}

After completing IMRT, patients returned for follow-up appointments at least every 3 months in the first three years and every 6 months thereafter (or until death). Follow-up was measured from first day of therapy to day of last examination or death. Patients who achieved a complete response but developed recurrence at the primary site after completing IMRT were defined as suffering local recurrence. Conventional examinations during follow-up were similar to the pretreatment assessment. Local relapse was diagnosed via MRI of the nasopharynx, biopsy, or both; PET-CT imaging was used when necessary. The primary end-point was LRFS, calculated from first day of therapy to date of local failure. Patients whose attendance at recent follow-up examinations was not recorded in their medical records were followed up by telephone.

\section{Statistical analysis}

This study aimed to establish an effective prognostic nomogram for individualized prediction of local recurrence and survival. SPSS ver. 22.0 (IBM Corp., Armonk, NY) was used to identify independent prognostic factors for LRFS. Forty-three candidate predictors were included in univariable analysis: age, sex, WHO pathologic type, N category, family history of NPC, smoking, pretreatment hemoglobin, body mass index (BMI), pretreatment viral capsid antigen IgA, pretreatment early antigen IgA, pretreatment EpsteinBarr virus DNA, pretreatment $\mathrm{LDH}$; cumulative dose to the GTVp; chemotherapy; pretreatment white blood cell (WBC) count; pretreatment neutrophil (Neu) count; pretreatment $\mathrm{Neu} /$ WBC ratio; pretreatment C-reactive protein; GTVp; prolonged radiation treatment; nasal cavity invasion; oropharyngeal invasion; parapharyngeal space invasion; medial pterygoid invasion; lateral pterygoid invasion; prevertebral muscle invasion; cervical vertebrae invasion; maxillary sinus invasion; frontal sinus invasion; sphenoid sinus invasion; ethmoidal sinus invasion; pterygopalatine fossa invasion; foramen lacerum invasion; foramen ovale invasion; clivus invasion; petrous apex invasion; great wing of sphenoid bone invasion; base of sphenoid bone invasion; cranial nerve invasion; intracranial invasion; orbit invasion; hypopharynx invasion and extensive soft tissue involvement (beyond the lateral surface of the lateral pterygoid muscle, parotid gland).

LRFS curves were plotted using the Kaplan-Meier method. Univariate and multivariate analysis were performed via Cox proportional hazard regression. Variables achieving significance $(p<0.05)$ were included in the Cox regression model with backward elimination for multivariate analyses.

A nomogram based on the results of the multivariate analysis was formulated to provide visualized risk prediction using the rms package of R ver. 3.2.5 (https://www. r-project.org/ ). A final model was selected using a backward stepdown process that incorporated Akaike's information criterion [15]. Nomograms were generated for the probabilities of local recurrence at 36 and 60 months.

The predictive accuracy of the nomogram was assessed by comparing discrimination and calibration. Predictive accuracy was assessed over 1,000 bootstrap replicates $[10,11]$. The discriminatory performance of the nomograms was measured using the concordance index (C-index) by comparing nomogram-predicted versus observed Kaplan-Meier estimates of survival probability, a variable equivalent to the area under curve of receiver operating characteristic curves for censored data. Maximum C-index (1.0) indicates a perfect match between predicted and actual probabilities, while 0.5 indicates the random chance of the model correctly predicting outcomes. Calibration of the nomograms for 3- and 5-year LRFS was performed using a calibration curve incor- 
Table 1. Clinical characteristics and treatment parameters of 415 patients with $\mathrm{T} 4$ nasopharyngeal carcinoma and association with local recurrence-free survival in univariate analysis

\begin{tabular}{|c|c|c|}
\hline Characteristic & No. of patients $(\%)(n=415)$ & p-value ${ }^{a}$ \\
\hline \multicolumn{3}{|l|}{ Age (yr) } \\
\hline$\leq 47$ & $216(52.0)$ & 0.011 \\
\hline$>47$ & $199(48.0)$ & \\
\hline \multicolumn{3}{|l|}{ Sex } \\
\hline Male & $328(79)$ & 0.659 \\
\hline Female & $87(21)$ & \\
\hline \multicolumn{3}{|l|}{ WHO pathology } \\
\hline Type I & $5(1.2)$ & 0.168 \\
\hline Type II & $15(3.6)$ & \\
\hline Type III & $395(95.2)$ & \\
\hline \multicolumn{3}{|l|}{$\mathbf{N}$ category $^{\mathrm{b})}$} \\
\hline No & $46(11.1)$ & 0.589 \\
\hline N1 & $262(63.1)$ & \\
\hline N2 & $70(16.9)$ & \\
\hline N3 & $37(8.9)$ & \\
\hline \multicolumn{3}{|l|}{ Family history of NPC } \\
\hline Yes & $38(9.2)$ & 0.628 \\
\hline No & $377(90.8)$ & \\
\hline \multicolumn{3}{|l|}{ Smoking } \\
\hline Yes & $180(43.4)$ & 0.985 \\
\hline No & $235(56.6)$ & \\
\hline \multicolumn{3}{|l|}{$\mathrm{Hb}(\mathrm{g} / \mathrm{L})$} \\
\hline$<113$ & $22(5.3)$ & 0.402 \\
\hline $113-151$ & $289(69.6)$ & \\
\hline$>151$ & $104(25.1)$ & \\
\hline \multicolumn{3}{|l|}{ BMI $\left(\mathrm{kg} / \mathrm{m}^{2}\right)$} \\
\hline$<22.2$ & $204(49.2)$ & 0.003 \\
\hline$\geq 22.2$ & $211(50.8)$ & \\
\hline \multicolumn{3}{|l|}{ VCA-IgA } \\
\hline$<1: 80$ & $65(15.7)$ & 0.354 \\
\hline 1:80-1:320 & $255(61.4)$ & \\
\hline$\geq 1: 640$ & $95(22.9)$ & \\
\hline \multicolumn{3}{|l|}{ EA-IgA } \\
\hline$<1: 10$ & $103(24.8)$ & 0.181 \\
\hline $1: 10-1: 20$ & $139(33.5)$ & \\
\hline$\geq 1: 40$ & $173(41.7)$ & \\
\hline \multicolumn{3}{|l|}{ EBV-DNA (copy/mL) } \\
\hline$<11,600$ & $206(49.6)$ & 0.245 \\
\hline$\geq 11,600$ & $209(50.4)$ & \\
\hline \multicolumn{3}{|l|}{ LDH (U/L) } \\
\hline$<245$ & $370(89.2)$ & 0.564 \\
\hline$\geq 245$ & $39(9.4)$ & \\
\hline \multicolumn{3}{|c|}{ Cumulative dose to GTVp (Gy) } \\
\hline$<68$ & $195(47.0)$ & 0.430 \\
\hline$\geq 68$ & $219(52.8)$ & \\
\hline \multicolumn{3}{|l|}{ Chemotherapy } \\
\hline None & $7(1.7)$ & 0.066 \\
\hline Concurrent & $104(25.1)$ & \\
\hline Neoadjuvant \pm concurrent & $304(73.3)$ & \\
\hline
\end{tabular}

(Continued to the next page) 
Table 1. Continued

\begin{tabular}{|c|c|c|}
\hline Characteristic & No. of patients $(\%)(n=415)$ & p-value ${ }^{a}$ \\
\hline \multicolumn{3}{|l|}{ WBC $\left(\times 10^{9} / \mathrm{L}\right)$} \\
\hline$<4$ & $14(3.4)$ & 0.135 \\
\hline $4-0$ & $345(83.1)$ & \\
\hline$\geq 10$ & $56(13.5)$ & \\
\hline \multicolumn{3}{|l|}{ Neu $\left(\times 10^{9} / \mathrm{L}\right)$} \\
\hline$<2$ & $17(4.1)$ & 0.048 \\
\hline $2-7$ & $334(80.5)$ & \\
\hline$\geq 7$ & $64(15.4)$ & \\
\hline \multicolumn{3}{|l|}{ Neu/WBC } \\
\hline$<0.7$ & $289(69.6)$ & 0.819 \\
\hline$\geq 0.7$ & $126(30.4)$ & \\
\hline \multicolumn{3}{|l|}{ CRP (g/mL) } \\
\hline$<1$ & $92(22.2)$ & 0.336 \\
\hline $1-3$ & $127(30.6)$ & \\
\hline$\geq 3$ & $196(47.2)$ & \\
\hline \multicolumn{3}{|l|}{ GTVp $\left(\mathrm{cm}^{3}\right)$} \\
\hline$<65.7$ & $207(49.9)$ & 0.002 \\
\hline$\geq 65.7$ & $208(50.1)$ & \\
\hline \multicolumn{3}{|c|}{ Prolonged radiation treatment, total days } \\
\hline No & $192(46.3)$ & 0.644 \\
\hline Yes & $223(53.7)$ & \\
\hline
\end{tabular}

WHO, World Health Organization; NPC, nasopharyngeal carcinoma; Hb, hemoglobin; BMI, body mass index; VCA-IgA, viral capsid antigen IgA; EA-IgA, pretreatment early antigen IgA; EBV, Epstein-Barr virus; $\mathrm{LDH}$, pretreatment lactate dehydrogenase; GTVp, primary gross tumor volume; WBC, white blood cells; Neu, neutrophils; CRP, C-reaction protein. ${ }^{a}$ p-values were

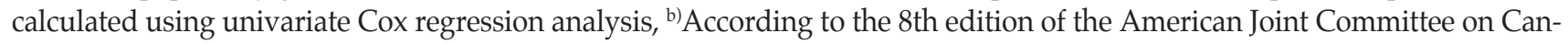
cer/Union for International Cancer Control staging system.

porating model-predicted and actual observed probabilities. Biases of predictive performance were first estimated using bootstrap sampling, then corrected with apparent measures to produce bias-corrected values to avoid overfitting [12]. Patients were classified into three risk subgroups for local recurrence based on the scores calculated by the nomograms using X-tile software [16]. Two-tailed p-values $<0.05$ were considered significant.

\section{Ethical statement}

This study was approved by our institutional review board (approve number: YB2017-032). As this was a retrospective analysis of routine clinical data, a waiver of the requirement for individual informed consent was granted by the institutional ethics committee.

\section{Results}

\section{Baseline characteristics, patterns of treatment failure, and survival}

Median follow-up for the whole cohort was 59.8 months (range, 3.3 to 84.4 months); median age was 47 years (range, 17 to 78 years). All 415 patients had T4N0-3M0 (stage IV) disease. Detailed clinicopathological characteristics and treatment factors are summarized in Table 1 . A total of 45/415 patients $(10.8 \%)$ developed local recurrence by last followup. The latency from the end of treatment to first recurrence ranged from 3.4 months to 85.6 months. Three- and 5-year LRFS were $91.6 \%$ and $88.8 \%$, respectively.

\section{Independent prognostic factors for LRFS}

Eleven factors including age, BMI, Neu, GTVp, orbit invasion, foramen lacerum invasion, maxillary sinus invasion, 
Table 2. Lesions in T1-T4 category structures among 415 patients with T4 nasopharyngeal carcinoma and association with local recurrence-free survival in univariate analysis

\begin{tabular}{|c|c|c|}
\hline Structure & No. of patients $(\%)(\mathrm{n}=415)$ & p-value \\
\hline \multicolumn{3}{|c|}{ Coincidental lesions in T1 category structures } \\
\hline \multicolumn{3}{|c|}{ Nasal cavity invasion } \\
\hline No & $188(45.3)$ & 0.397 \\
\hline Unilateral & $145(34.9)$ & \\
\hline Bilateral & $82(19.8)$ & \\
\hline \multicolumn{3}{|c|}{ Oropharyngeal invasion } \\
\hline No & $343(82.7)$ & 0.296 \\
\hline Unilateral & $60(14.5)$ & \\
\hline Bilateral & $12(2.9)$ & \\
\hline \multicolumn{3}{|c|}{ Coincidental lesions in T2 category structures } \\
\hline \multicolumn{3}{|c|}{ Parapharyngeal space invasion } \\
\hline No & $50(12.0)$ & 0.638 \\
\hline Unilateral & $252(60.8)$ & \\
\hline Bilateral & $113(27.2)$ & \\
\hline \multicolumn{3}{|c|}{ Medial pterygoid invasion } \\
\hline No & $152(36.6)$ & 0.385 \\
\hline Unilateral & $235(56.6)$ & \\
\hline Bilateral & $28(6.7)$ & \\
\hline \multicolumn{3}{|c|}{ Lateral pterygoid invasion } \\
\hline No & $286(68.9)$ & 0.499 \\
\hline Unilateral & $124(29.9)$ & \\
\hline Bilateral & $5(1.2)$ & \\
\hline \multicolumn{3}{|c|}{ Prevertebral muscle } \\
\hline No & $83(20.0)$ & 0.122 \\
\hline Unilateral & $189(45.5)$ & \\
\hline Bilateral & $143(34.5)$ & \\
\hline \multicolumn{3}{|c|}{ Coincidental lesions in T3 categories structures } \\
\hline \multicolumn{3}{|c|}{ Cervival vertebrae invasion } \\
\hline No & $400(96.4)$ & 0.410 \\
\hline Unilateral & $13(3.1)$ & \\
\hline Bilateral & $2(0.5)$ & \\
\hline \multicolumn{3}{|c|}{ Maxillary sinus invasion } \\
\hline No & $351(84.6)$ & 0.007 \\
\hline Unilateral & $54(13.0)$ & \\
\hline Bilateral & $10(2.4)$ & \\
\hline \multicolumn{3}{|c|}{ Frontal sinus invasion } \\
\hline No & $414(99.8)$ & 0.825 \\
\hline Unilateral & $1(0.2)$ & \\
\hline Bilateral & 0 & \\
\hline \multicolumn{3}{|c|}{ Sphenoid sinus invasion } \\
\hline No & $196(47.2)$ & 0.076 \\
\hline Unilateral & $218(52.5)$ & \\
\hline Bilateral & $1(0.2)$ & \\
\hline \multicolumn{3}{|c|}{ Ethmoidal sinus invasion } \\
\hline No & 339 (81.7) & $<0.001$ \\
\hline Unilateral & 43 (10.4) & \\
\hline Bilateral & $33(8.0)$ & \\
\hline
\end{tabular}

(Continued to the next page) 
Table 2. Continued

Pterygopalatine fossa invasion

No

Unilateral

$194(46.7)$

$180(43.4)$

Bilateral

$41(9.9)$

Foramen lacerum invasion

No

Unilateral

Bilateral

Foramen ovale invasion

No

Unilateral

Bilateral

Clivus invasion

No

Unilateral

Bilateral

Petrous apex invasion

No

Unilateral

Bilateral

Great wing of sphenoid bone invasion

No

Unilateral

Bilateral

Base of sphenoid bone invasion

No

Unilateral

Bilateral

\section{Coincidental lesions in T4 categories structures}

Cranial nerve invasion

Yes

No

Intracranial invasion

No

Unilateral

Bilateral

Orbit invasion

No

Unilateral

Bilateral

Hypopharynx invsaion

No

Unilateral

Bilateral

Extensive soft tissue invasion (beyond lateral surface of lateral pterygoid muscle, parotid gland)

No

Unilateral

Bilateral
346 (83.4)

67 (16.1)

73 (17.6)

264 (63.6)

78 (18.8)

169 (40.7)

$226(54.5)$

$20(4.8)$

$36(8.7)$

$378(91.1)$

$1(0.2)$

43 (10.4)

255 (61.4)

117 (28.2)

175 (42.2)

209 (50.4)

31 (7.5)

28 (6.7)

384 (92.6)

$3(0.7)$

$137(33.0)$

$278(67.0)$

74 (17.8)

303 (73.0)

$38(9.2)$

356 (85.8)

54 (13.0)

5 (1.2)

393 (94.7)

16 (3.9)

$6(1.4)$

$2(0.5)$
0.066

0.013

0.069

0.246

0.004

0.041

0.095

0.457

0.032

0.019

0.159

p-values were calculated using univariate Cox regression analysis. 
Table 3. Summary of multivariate analysis of independent prognostic factors for LRFS in T4 nasopharyngeal carcinoma

\begin{tabular}{|c|c|c|c|}
\hline \multirow{2}{*}{ Characteristic } & \multicolumn{3}{|c|}{ LRFS } \\
\hline & p-value ${ }^{a)}$ & HR & $95 \% \mathrm{CI}$ \\
\hline \multicolumn{4}{|c|}{ Ethmoidal sinus invasion } \\
\hline No & Reference & & \\
\hline Unilateral & 0.087 & 1.999 & $0.905-4.415$ \\
\hline Bilateral & 0.010 & 2.798 & $1.285-6.090$ \\
\hline GTVp $\left(\mathrm{cm}^{3}\right)$ & 0.015 & 2.277 & $1.170-4.430$ \\
\hline \multicolumn{4}{|l|}{$<65.7$} \\
\hline \multicolumn{4}{|l|}{$\geq 65.7$} \\
\hline Age (yr) & 0.010 & 2.243 & $1.215-4.142$ \\
\hline \multicolumn{4}{|l|}{$\leq 47$} \\
\hline \multicolumn{4}{|l|}{$>47$} \\
\hline BMI $\left(\mathrm{kg} / \mathrm{m}^{2}\right)$ & 0.020 & 0.459 & $0.239-0.883$ \\
\hline \multicolumn{4}{|l|}{$<22.2$} \\
\hline$\geq 22.2$ & & & \\
\hline
\end{tabular}

LRFS, local relapse-free survival; HR, hazard ratio; CI, confidence interval; GTVp, primary gross tumor volume; BMI, body mass index. ${ }^{a)}$ p-values were calculated using the Cox proportional hazards model.

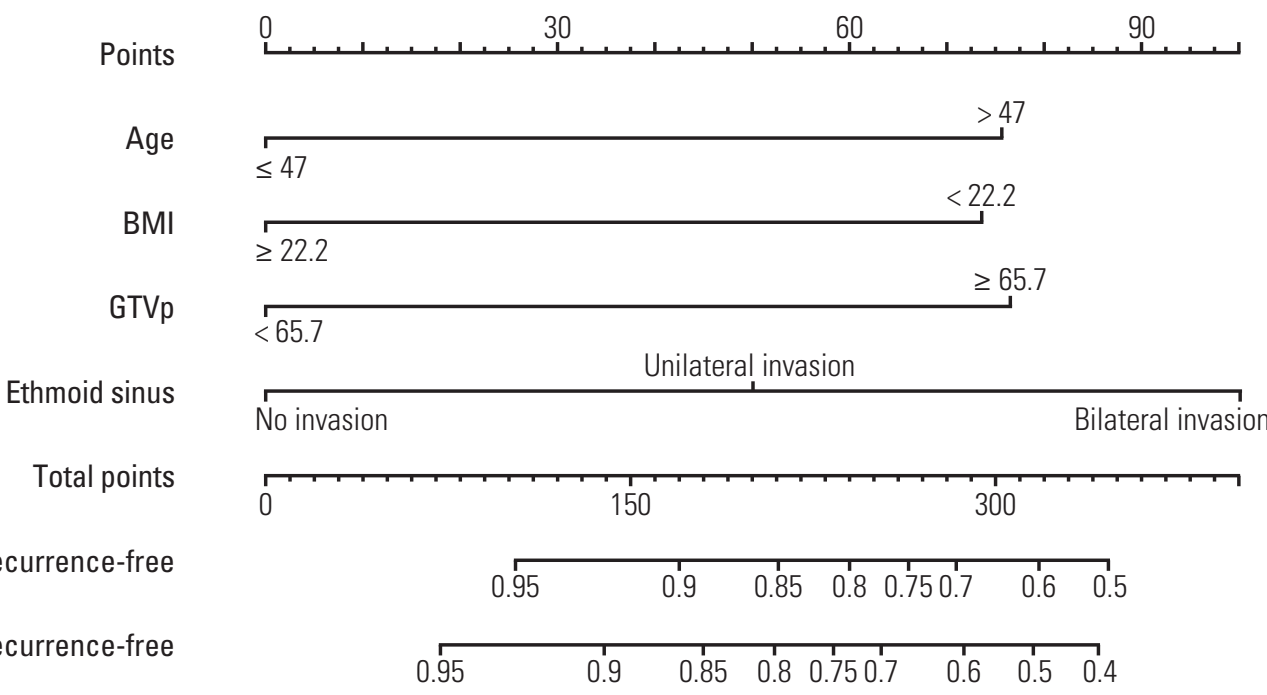

Fig. 1. Nomogram for local recurrence in non-metastatic T4 nasopharyngeal carcinoma after radical intensity-modulated radiation therapy. BMI, body mass index; GTVp, primary gross tumor volume.

intracranial invasion, ethmoidal sinus invasion, petrous apex invasion, and invasion of the great wing of the sphenoid bone were significantly associated with local recurrence in univariate analysis (Tables 1 and 2).

The significant 11 factors from univariate analysis were included in Cox multivariate analysis of LRFS to adjust for various prognostic factors. Ethmoidal sinus invasion, GTVp, age, and pretreatment BMI were independent prognostic factors for LRFS in multivariate analysis. Bilateral ethmoidal sinus invasion, large GTVp, old age, and low BMI were significantly associated with poorer LRFS (Table 3). 
A

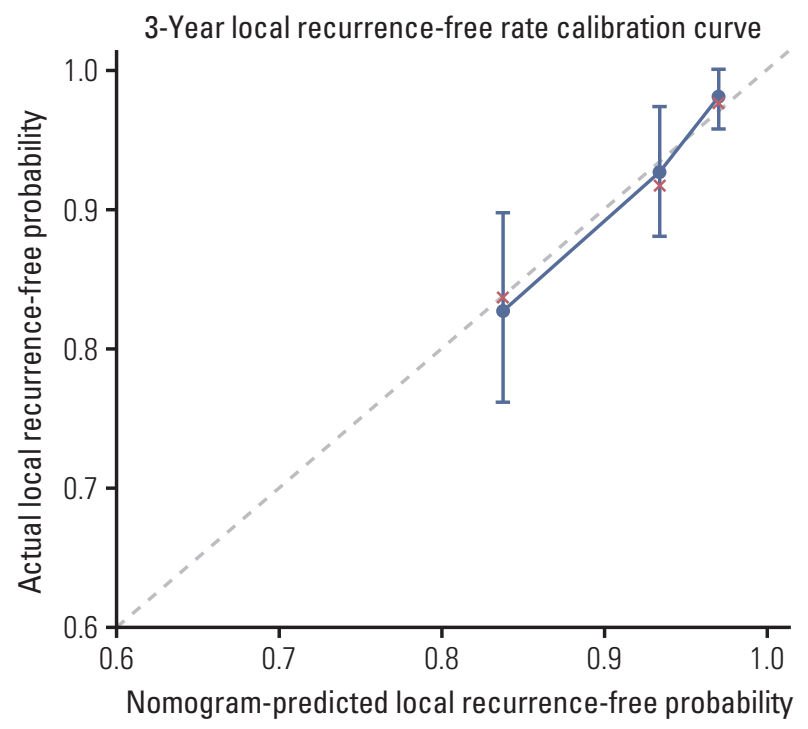

B

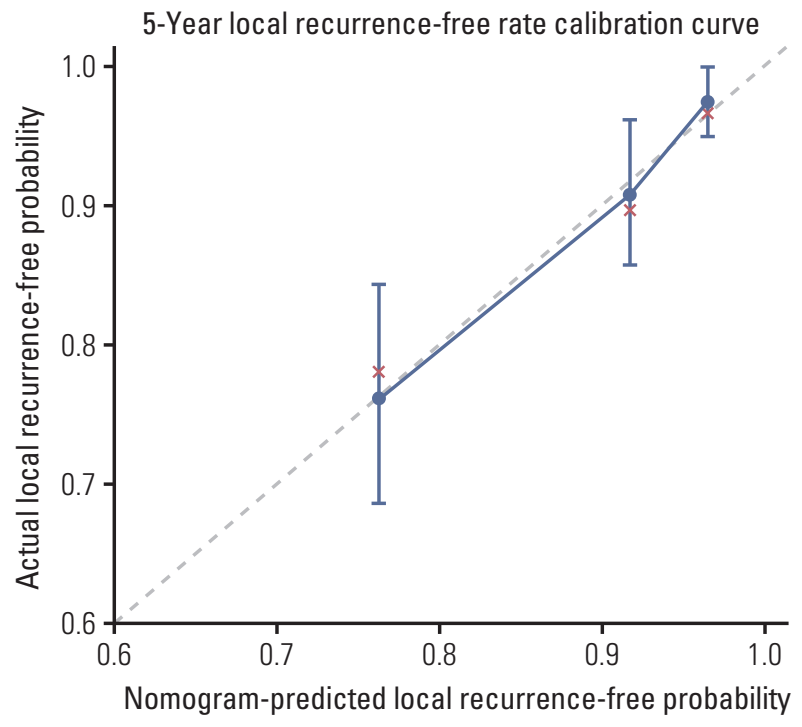

Fig. 2. Calibration curves for predicting local recurrence at 3 years (A) and 5 years (B). Nomogram-predicted probability of local recurrence is plotted on the $\mathrm{x}$-axis; actual observed local recurrence-free probability is plotted on the y-axis.

\section{Development and internal validation of a nomogram for local recurrence}

The prognostic nomogram that incorporates all significant independent factors for LRFS is presented in Fig. 1. The values for each of the model covariates were mapped to points on a scale axis ranging from 0 to 100 . By adding up the total score for all variables and locating this value on the total point scale, the probability of local recurrence can be determined by drawing a vertical line to the total score. The nomogram indicates ethmoid sinus involvement has the most significant effect on LRFS, followed by GTVp, age and BMI. In Fig. 2, the x-axes denote the predicted probabilities of LRFS from the nomograms, the y-axes denote actual probabilities of LRFS calculated using the Kaplan-Meier method, and the 45-degree line represents the ideal reference line where nomogram-predicted and observed LRFS probabilities are exactly the same. The calibration plots indicated excellent agreement between nomogram-predicted LRFS and actual 3- and 5-year LRFS probabilities. The c-index of the nomogram for local recurrence was 0.732 (95\% confidence interval [CI], 0.726 to 0.738 ), demonstrating the nomogram represents a reasonable model for predicting local recurrence.

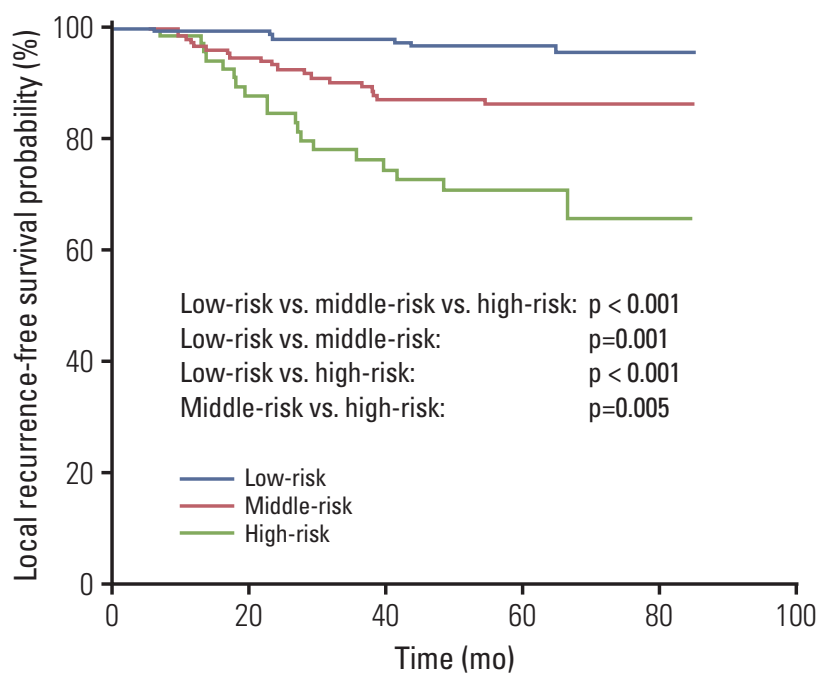

Fig. 3. Kaplan-Meier local recurrence-free survival curves for patients with T4 nasopharyngeal carcinoma based on risk group stratification. Patients were classified into a low-risk group (score < 123), intermediate-risk group (123202), and high-risk group (203-325) using total nomogram point score cutoff values determined using X-tile software. 


\section{Stratification of the risk of local recurrence for patients with T4 NPC}

Based on the cutoff values of the total point scores for local recurrence determined using $\mathrm{X}$-tile software, patients were classified into a low-risk group (score $<123$ ), intermediaterisk group (123-202), and high-risk group (203-325). In summary, a total of $193(46.5 \%), 152(36.5 \%)$, and $70(16.9 \%)$ patients were assigned to the low-, intermediate-, and highrisk groups, respectively. Three-year LRFS for the low-, intermediate-, and high-risk groups was $97.9 \%, 90.1 \%$, and $76.2 \%$, respectively; and 5-year LRFS was $96.7 \%, 86.2 \%$ and $70.8 \%$, respectively. Kaplan-Meier survival analysis demonstrated this risk stratification effectively discriminated the risk of LRFS in patients with T4 NPC treated with IMRT $(\mathrm{p}<0.001)$ (Fig. 3).

\section{Discussion}

$\mathrm{T}$ category is not the sole risk factor for local recurrence in NPC: the LRFS rates of patients with the same T category can vary widely [5]. Thus, T category is not capable of accurately predicting local recurrence in patients with NPC treated with IMRT. Furthermore, while previous research demonstrated no significant differences in LRFS between patients with T1, T2, and T3 NPC; patients with T4 disease have a significantly poorer local control rate [5]. Establishing a prognostic nomogram for each $\mathrm{T}$ category may help to provide more individualized and accurate estimation of the risk of local recurrence following IMRT; this would be especially significant for patients with T4 NPC who have the highest risk of local recurrence. More importantly, analysis of the risk of local relapse in T4 NPC is crucial for formulation of appropriate treatment approaches; a nomogram may enable clinicians to develop more individualized multidisciplinary treatment and follow-up strategies.

To the best of our knowledge, this is the first study to develop a prognostic nomogram for LRFS based on widelyassessed clinicopathological factors in patients with nonmetastatic T4 NPC treated with definitive IMRT. The multivariable Cox analyses demonstrated ethmoidal sinus invasion, GTVp, age and BMI were independent prognostic factors for LRFS in non-metastatic T4 NPC. The resulting nonograms demonstrated excellent discriminative ability (0.732; 95\% CI, 0.726 to 0.738$)$ with excellent agreement between nomogram-predicted LRFS and actual LRFS probabilities, as confirmed by the calibration curve. In addition, the nomogram-generated scores enabled patients with T4 NPC to be further stratified into three distinct risk groups for
LRFS, and as expected, the proposed risk groups significantly discriminated the risk of LRFS within T4 NPC, especially for the high-risk group. Thus, this nomogram may represent a clinically useful tool to supplement the traditional TNM staging system.

The nomogram revealed ethmoid sinus involvement had the most significant association with LRFS, followed by GTVp, age, and BMI. Consistent with numerous previous studies, GTV-p was a significant prognostic factor for LRFS in T4 NPC after IMRT $[6,7,17,18]$. A large GTVp was associated with poorer LRFS; the following factors may explain this phenomenon. First, a large GTVp, which reflects a high tumor burden, often indicates tumor hypoxia. Tumor hypoxia plays a key role in the development of radio- and chemoresistance, and promotes disease progression such as local recurrence [19-22]. Secondly, a large GTVp indicates the primary tumor is closer to critical normal organs at risk, which negatively affects local radiation dose escalation to the involved areas.

However, Sakata et al. [23] reported that deep primary tumor infiltration was a more important adverse prognostic factor than a large GTVp. we found both ethmoid sinus invasion and a large GTVp were adverse independent prognostic factors for local recurrence in T4 NPC, with ethmoid sinus invasion having a greater contribution than GTVp to local recurrence. The ethmoid sinus is located distant to the nasopharynx and is a low-risk site of invasion in NPC. After lesions in various anatomical structures belonging to the T1-T4 categories were incorporated into the analysis, we found that only ethmoid sinus invasion was an adverse independent prognostic factor for local recurrence in T4 NPC. In fact, deep infiltration of the ethmoid sinus is a marker of severe extension; therefore, ethmoid sinus invasion is inherently associated a larger GTVp.

$\mathrm{BMI}$ and age were also significant prognostic factors for local recurrence in T4 NPC; older age (> 47-years-old) and lower BMI $\left(<22.2 \mathrm{~kg} / \mathrm{m}^{2}\right)$ were associated with poor LRFS. Consistent with these results, Cheng et al. [9] showed older age (> 40-years-old) adversely affected locoregional control in NPC after definitive conventional radiotherapy. Moreover, Tang et al. [24] demonstrated low pretreatment BMI was associated with local and systemic recurrence. It is plausible that lower BMI and older age may be associated with poorer immune system function and reduced tolerance to intensive treatment, which may ultimately result poorer survival.

The nomogram established in the present study has some limitations. First, this was a retrospective analysis of medical records; therefore, selection bias may exist. However, the relatively large sample size should reduce the influence of such bias. Secondly, the nomogram was not subject to external verification due to the lack of an external validation cohort. 
However, we conducted internal validation via 1,000 bootstrap replicates to avoid over-fitting of the data. In summary, the nomogram developed in this study may enable clinicians to make accurate predictions of LRFS for patients with T4 NPC after definitive IMRT. We hope the nomograms could be applied to aid patient counseling or guide individual treatment, which may help to further improve local control. The prognostic value offered by this pretreatment nomogram for predicting local recurrence could enable more aggressive treatment protocols to be delivered to patients at high-risk of local recurrence. Concurrent chemoradiotherapy (CCRT) is now recommended as a standard treatment for T4N0-3M0 NPC. However, induction chemotherapy has been shown to reduce the risk of locoregional recurrence [25]. Thus, induction chemotherapy combined with CCRT may be able to achieve an improvement in local control for patients with $\mathrm{T} 4$ NPC, although further evidence is needed to prove this hypothesis. Additional studies are required to further validate this nomogram in other patient cohorts.

\section{Conflicts of Interest}

Conflict of interest relevant to this article was not reported.

\section{Acknowledgments}

This work was supported by grants from the Health \& Medical Collaborative Innovation Project of Guangzhou City, China (201604020003), the Planned Science and Technology Project of Guangdong Province (20160307), the Special Support Program of Sun Yat-sen University Cancer Center, and the Sun Yat-Sen University Cultivating Project and Interdisciplinary Research Grant.

\section{Author Details}

${ }^{1}$ Department of Radiation Oncology, Sun Yat-sen University Cancer Center, State Key Laboratory of Oncology in South China, Collaborative Innovation Center for Cancer Medicine, Guangzhou, ${ }^{2}$ Department of Oncology, the First Affiliated Hospital of Guangdong Pharmaceutical University, Guangdong, ${ }^{3}$ Department of Medical Statistics and Epidemiology, School of Public Health, Sun Yat-sen University, Guangzhou, China

\section{References}

1. Jemal A, Bray F, Center MM, Ferlay J, Ward E, Forman D. Global cancer statistics. CA Cancer J Clin. 2011;61:69-90.

2. Chan AT. Nasopharyngeal carcinoma. Ann Oncol. 2010;21 Suppl 7:vii308-12.

3. Lee AW, Lin JC, Ng WT. Current management of nasopharyngeal cancer. Semin Radiat Oncol. 2012;22:233-44.

4. Lai SZ, Li WF, Chen L, Luo W, Chen YY, Liu LZ, et al. How does intensity-modulated radiotherapy versus conventional two-dimensional radiotherapy influence the treatment results in nasopharyngeal carcinoma patients? Int J Radiat Oncol Biol Phys. 2011;80:661-8.

5. Wu LR, Liu YT, Jiang N, Fan YX, Wen J, Huang SF, et al. Tenyear survival outcomes for patients with nasopharyngeal carcinoma receiving intensity-modulated radiotherapy: an analysis of 614 patients from a single center. Oral Oncol. 2017;69:26-32.

6. Xu T, Tang J, Gu M, Liu L, Wei W, Yang H. Recurrent nasopharyngeal carcinoma: a clinical dilemma and challenge. Curr Oncol. 2013;20:e406-19.

7. Li JX, Huang SM, Jiang XH, Ouyang B, Han F, Liu S, et al. Local failure patterns for patients with nasopharyngeal carcinoma after intensity-modulated radiotherapy. Radiat Oncol. 2014;9:87.

8. Wan XB, Wei L, Li H, Dong M, Lin Q, Ma XK, et al. High pretreatment serum lactate dehydrogenase level correlates with disease relapse and predicts an inferior outcome in locally advanced nasopharyngeal carcinoma. Eur J Cancer. 2013;49: 2356-64.

9. Cheng SH, Tsai SY, Horng CF, Yen KL, Jian JJ, Chan KY, et al. A prognostic scoring system for locoregional control in nasopharyngeal carcinoma following conformal radiotherapy. Int J Radiat Oncol Biol Phys. 2006;66:992-1003.

10. Balachandran VP, Gonen M, Smith JJ, DeMatteo RP. Nomograms in oncology: more than meets the eye. Lancet Oncol. 2015;16:e173-80.

11. Iasonos A, Schrag D, Raj GV, Panageas KS. How to build and interpret a nomogram for cancer prognosis. J Clin Oncol. 2008;26:1364-70.

12. Bendifallah S, Ballester M, Uzan C, Fauvet R, Morice P, Darai E. Nomogram to predict recurrence in patients with early- and advanced-stage mucinous and serous borderline ovarian tumors. Am J Obstet Gynecol. 2014;211:637.e1-6.

13. Li WF, Sun Y, Chen M, Tang LL, Liu LZ, Mao YP, et al. Locoregional extension patterns of nasopharyngeal carcinoma and suggestions for clinical target volume delineation. Chin J Cancer. 2012;31:579-87.

14. Liang SB, Sun Y, Liu LZ, Chen Y, Chen L, Mao YP, et al. Extension of local disease in nasopharyngeal carcinoma detected by magnetic resonance imaging: improvement of clinical target volume delineation. Int J Radiat Oncol Biol Phys. 
2009;75:742-50.

15. Harrell FE Jr, Lee KL, Mark DB. Multivariable prognostic models: issues in developing models, evaluating assumptions and adequacy, and measuring and reducing errors. Stat Med. 1996;15:361-87.

16. Camp RL, Dolled-Filhart M, Rimm DL. X-tile: a new bioinformatics tool for biomarker assessment and outcome-based cut-point optimization. Clin Cancer Res. 2004;10:7252-9.

17. Ng WT, Lee MC, Chang AT, Chan OS, Chan LL, Cheung FY, et al. The impact of dosimetric inadequacy on treatment outcome of nasopharyngeal carcinoma with IMRT. Oral Oncol. 2014;50:506-12.

18. Tao CJ, Liu X, Tang LL, Mao YP, Chen L, Li WF, et al. Prognostic scoring system for locoregional control among the patients with nasopharyngeal carcinoma treated by intensitymodulated radiotherapy. Chin J Cancer. 2013;32:494-501.

19. Kong F, Ying H, Du C, Huang S, Zhou J, Chen J, et al. Patterns of local-regional failure after primary intensity modulated radiotherapy for nasopharyngeal carcinoma. Radiat Oncol. 2014;9:60.

20. Hong B, Lui VW, Hashiguchi M, Hui EP, Chan AT. Targeting tumor hypoxia in nasopharyngeal carcinoma. Head Neck. 2013;35:133-45.
21. Overgaard J. Hypoxic radiosensitization: adored and ignored. J Clin Oncol. 2007;25:4066-74.

22. Cosse JP, Michiels C. Tumour hypoxia affects the responsiveness of cancer cells to chemotherapy and promotes cancer progression. Anticancer Agents Med Chem. 2008;8:790-7.

23. Sakata K, Hareyama M, Tamakawa M, Oouchi A, Sido M, Nagakura H, et al. Prognostic factors of nasopharynx tumors investigated by MR imaging and the value of MR imaging in the newly published TNM staging. Int J Radiat Oncol Biol Phys. 1999;43:273-8.

24. Tang LQ, Li CF, Li J, Chen WH, Chen QY, Yuan LX, et al. Establishment and validation of prognostic nomograms for endemic nasopharyngeal carcinoma. J Natl Cancer Inst. 2016;108:djv291.

25. Chua DT, Sham JS, Choy D, Lorvidhaya V, Sumitsawan Y, Thongprasert $S$, et al. Preliminary report of the Asian-Oceanian Clinical Oncology Association randomized trial comparing cisplatin and epirubicin followed by radiotherapy versus radiotherapy alone in the treatment of patients with locoregionally advanced nasopharyngeal carcinoma. Asian-Oceanian Clinical Oncology Association Nasopharynx Cancer Study Group. Cancer. 1998;83:2270-83. 